\title{
A GAUGE-FIELD APPROACH TO 3- AND 4-MANIFOLD INVARIANTS
}

\author{
BOGUSEAW BRODA \\ Department of Theoretical Physics, University of Eódź \\ Pomorska 149/153, PL-90-236 Eódź, Poland \\ E-mail: bobroda@mvii.uni.lodz.pl
}

\begin{abstract}
An approach to construction of topological invariants of the Reshetikhin-TuraevWitten type of 3- and 4-dimensional manifolds in the framework of SU(2) Chern-Simons gauge theory and its hidden (quantum) gauge symmetry is presented.
\end{abstract}

1. Introduction. The issue of topological classification of low-dimensional manifolds, especially of dimensions 3 and 4 (the most difficult and interesting ones), is a challenging problem in modern mathematics. One of the most spectacular events in topology of 3 -dimensional manifolds took place a few years ago, when a new (numerical) topological invariant of closed orientable 3-manifolds, parametrized by the integer $k$, defined via surgery on a framed link, was discovered. The idea is due to a physicist, Edward Witten, who proposed the invariant in his famous paper on quantum field theory and the Jones polynomial [Wit1]. The first explicit and rigorous construction is due to mathematicians, Reshetikhin and Turaev [RT]. Their approach is combinatorial, whereas noncombinatorial possibilities, very straightforward though mathematically less rigorous, are offered by topological quantum field theory. The 3-dimensional invariant, known as the Reshetikhin-Turaev-Witten (RTW) invariant, is also frequently referred to as the $\mathrm{SU}(2)$ invariant because the Kauffman bracket it bases upon (denoted in mathematical literature as ' \langle\rangle ') formally corresponds, in Witten's approach, to the average with respect to the connection $A$ (defined on a trivial SU(2) bundle on the 3-manifold $M$ ) modulo gauge transformations, weighted by $\exp \left[i k S_{\mathrm{CS}}(A)\right]$. Here $S_{\mathrm{CS}}(A)$ is the Chern-Simons secondary characteristic class. Incidentally, the average is also denoted as ' \langle\rangle '. The construction of the RTW invariant makes use of the fundamental theorem of surgery of Lickorish and Wallace on presentation of every closed connected orientable 3-manifold $M$ via surgery

1991 Mathematics Subject Classification: Primary 57R65; Secondary 81T13.

Research supported by the KBN grants 2P30213906, 2P30221706p01, and the University of Łódź grant 505/457.

The paper is in final form and no version of it will be published elsewhere. 
on a framed link in $\mathcal{S}^{3}$, and the linear skein theory associated with the Kauffman bracket. The construction of the RTW invariant amounts to showing its invariance with respect to the Kirby moves $\left(\mathrm{K}_{1}\right.$ and $\left.\mathrm{K}_{2}\right)$. The Kirby moves are the allowable moves on a framed link, changing in general (isotopy class of) the link but not changing the 3 -manifold $M$ obtained from the link. In fact, $\mathrm{K}_{2}$ does not even change the 4-manifold $\mathcal{M}(M=\partial \mathcal{M})$, because it corresponds to sliding handles, whereas $\mathrm{K}_{1}$ adds a complex projective space, which changes $\mathcal{M}$ but still it does not change the 3-dimensional boundary $M$. The Kirby move $K_{1}$ means that we can add an unknotted, unlinked component with framing \pm 1 , i.e. $\bigcirc_{ \pm 1}$, whereas $K_{2}$ amounting to sliding a (upper) line over a (lower) trivial unknot in an annulus (a bordered exterior of "•"), which is, in general, non-trivially immersed in $\mathcal{S}^{3}$ is schematically depicted as

$$
\bar{\odot} \lessdot
$$

In Section 2 we aim to propose a new, heuristic, non-combinatorial derivation of the RTW invariant in the framework of non-perturbative (topological) quantum ChernSimons (CS) gauge theory [Bro3]. The idea is extremely simple, and in principle it applies to an arbitrary compact (semi-) simple Lie group $G$ (not only to the $\mathrm{SU}(2)$ one). Our invariant is essentially the partition function of CS theory on the 3-manifold $M_{L}$, defined via surgery on the framed link $L$ in the 3 -dimensional sphere $\mathcal{S}^{3}$. Actually, surgery instructions are implemented in the most direct and literal way. The method of cutting and pasting back is explicitly used in the standard field-theoretical fashion. Roughly speaking, cutting corresponds to fixing, whereas pasting back to identification and summing up the boundary conditions.

Section 3 is devoted to a 4-dimensional generalization of the RTW invariant, and is mathematically more rigorous [Bro1]. In dimension 4, there is a celebrated theorem of Freedman on classification of closed orientable simply-connected 4-manifolds, provided by the intersection form $Q(\mathcal{M})$ (and the Kirby-Siebenman invariant $\alpha(\mathcal{M})$ ). The intersection form $Q(\mathcal{M})$ corresponds to, and for 4-manifolds with boundary defined via surgery on a link in $\mathcal{S}^{3}$ is equal to, the linking matrix $\ell k$. The elements of the symmetric matrix $\ell k$, the linking numbers (with framing numbers on the diagonal), are the simplest numerical invariants of a link. Therefore, one can ask the following questions. Can one use 'nonabelian' invariants of links, for example the Kauffman bracket polynomial, to obtain 'non-abelian' invariants of 4-dimensional manifolds? Can one extend the idea of RTW to the 4-dimensional case? Can one treat simply-connected and non-simply-connected manifolds uniquely? The answer to these questions seems to be affirmative. Namely, we propose an invariant of closed connected orientable 4-manifolds, defined via surgery on a special link in $\mathcal{S}^{3}$. Thus, we have found a quantity invariant with respect to the 4dimensional version of the 'Kirby moves'. The idea as well as the construction resembles the original one, proposed by RTW in the 3-dimensional case, whereas the 4-dimensional version of the Kirby calculus we need has been developed by César de Sá [CdS].

In Section 4, we mention some other known invariants of 3- and 4-dimensional manifolds. 
2. Gauge-field approach to 3-manifold invariants. Our principal goal is to compute the partition function $Z\left(M_{L}\right)$ of CS theory on the manifold $M_{L}$, defined via honest/integer surgery on the framed link $L=\bigcup_{i=1}^{N} K_{i}$ in $\mathcal{S}^{3}$ (i.e. attaching 2-handles to a 4-ball along a framed link - a particular case of rational surgery manipulating only tori), for the $\mathrm{SU}(2)$ (gauge) Lie group. Obviously, the starting point is the partition function [Wit1] of CS theory $Z\left(\mathcal{S}^{3}\right)$ on the sphere $\mathcal{S}^{3}$

$$
Z\left(\mathcal{S}^{3}\right)=\int e^{i k S_{\mathrm{CS}}(A)} \mathcal{D} A
$$

where the functional integration is performed with respect to the connections $A$ modulo gauge transformations, defined on a trivial $\mathrm{SU}(2)$ bundle on $\mathcal{S}^{3}$. The classical action is the CS secondary characteristic class

$$
S_{\mathrm{CS}}(A)=\frac{1}{4 \pi} \int_{\mathcal{S}^{3}} \operatorname{Tr}\left(A d A+\frac{2}{3} A^{3}\right),
$$

and the expectation value of an observable $\mathcal{O}$ is defined as

$$
\langle\mathcal{O}\rangle=\int \mathcal{O} e^{i k S_{\mathrm{CS}}(A)} \mathcal{D} A
$$

According to the surgery prescription [Rol], we should cut out a closed tubular neighborhood $N_{i}$ of $K_{i}$ (a solid torus), and paste back a copy of a solid torus $T$, matching the meridian of $T$ to the (twisted by framing number) longitude on the boundary torus $\partial N_{i}$ in $\mathcal{S}^{3}$. To this end, in the first step, we should fix boundary conditions for the field $A$ on the twisted longitude represented by $K_{i}$. Since the only gauge-invariant (modulo conjugation) quantity defined on a closed curve is holonomy, we associate the holonomy operator $\operatorname{Hol}_{K_{i}}(A)$ to each knot $K_{i}$. Thus the symbol

$$
Z\left(\mathcal{S}^{3}, L ; g_{1}, g_{2}, \ldots, g_{N}\right)
$$

should be understood as the constrained partition function of CS theory, i.e. the values of holonomies along $K_{i}$ are fixed

$$
\operatorname{Hol}_{K_{i}}(A)=g_{i}, \quad i=1,2, \ldots, N
$$

Now, we can put

$$
Z\left(\mathcal{S}^{3}, L ; g_{1}, g_{2}, \ldots, g_{N}\right)=\left\langle\prod_{i=1}^{N} \delta\left(g_{i}, \operatorname{Hol}_{K_{i}}(A)\right)\right\rangle,
$$

where $\delta$ is a (group-theoretic) Dirac delta-function. Its explicit form following from the (group-theoretic) Fourier expansion is

$$
\delta(g, h)=\sum_{n} \overline{\chi_{n}(g)} \chi_{n}(h)
$$

where $n$ labels inequivalent irreducible representations (irrep's) of $\mathrm{SU}(2)$, and $\chi_{n}$ is a character. Physical observables being used in CS theory are typically Wilson loops, defined as

$$
W_{n}^{K}(A)=\operatorname{Tr}_{n}\left(\operatorname{Hol}_{K}(A)\right) \equiv \chi_{n}\left(\operatorname{Hol}_{K}(A)\right) .
$$


By virtue of $(2-3)$

$$
\delta\left(g_{i}, \operatorname{Hol}_{K_{i}}(A)\right)=\sum_{n} \overline{\chi_{n}\left(g_{i}\right)} W_{n}^{K_{i}}(A)
$$

Inserting (4) into (1) yields, as a basic building block, the following representation of the constrained partition function

$$
Z\left(\mathcal{S}^{3}, L ; g_{1}, g_{2}, \ldots, g_{N}\right)=\left\langle\prod_{i=1}^{N} \sum_{n_{i}} \overline{\chi_{n_{i}}\left(g_{i}\right)} W_{n_{i}}^{K_{i}}(A)\right\rangle .
$$

In the second step of our construction, we should paste back the tori matching the pairs of "longitudes" (the twisted longitudes and the meridians), i. e. we should identify and sum up the boundary conditions. Since the interior of a solid torus is homeomorphic to $\mathcal{S}^{3}$ with a removed solid torus, actually the meridians play the role of longitudes in analogous cutting procedures for an unknot $\{\bigcirc\}$ (with reversed orientation). Thus the partition function of CS theory on $M_{L}$ is

$$
\begin{aligned}
Z\left(M_{L}\right)=\int \prod_{i=1}^{N} d g_{i} Z\left(\mathcal{S}^{3}, \bigcirc ; g_{i}^{-1}\right) Z\left(\mathcal{S}^{3}, L ; g_{1}, g_{2}, \ldots, g_{N}\right) \\
\quad=\int \prod_{i=1}^{N} d g_{i} \sum_{m_{i}} \sum_{n_{i}} \overline{\chi_{m_{i}}\left(g_{i}^{-1}\right)} \overline{\chi_{n_{i}}\left(g_{i}\right)}\left\langle W_{m_{i}}^{\bigcirc}(A)\right\rangle\left\langle\prod_{j=1}^{N} W_{n_{j}}^{K_{j}}(A)\right\rangle,
\end{aligned}
$$

where the reversed orientation of the unknots $\{\bigcirc\}$ (corresponding to the meridians of the pasted back tori) accounts for the power -1 of the group elements $g_{i}$. From the orthogonality relations for characters and unitarity of irrep's, it follows that the 3-manifold invariant is of the form

$$
Z\left(M_{L}\right)=\left\langle\prod_{i=1}^{N} \omega_{K_{i}}(A)\right\rangle
$$

where

$$
\omega_{K_{i}}(A) \equiv \sum_{n_{i}}\left\langle W_{n_{i}}^{\bigcirc}(A)\right\rangle W_{n_{i}}^{K_{i}}(A)
$$

is an element of the linear skein of an annulus, immersed in the plane as a regular neighborhood of $K_{i}$. $\left\langle W_{n}^{\bigcirc}(A)\right\rangle$ are some computable coefficients depending on $n$ and $k$. Eq. (5) can be easily generalized to accommodate an ordinary link $\mathcal{L}=\bigcup_{i=1}^{M} \mathcal{K}_{i}$ embedded in $M_{L}$

$$
\left\langle\prod_{i=1}^{M} W_{n_{i}}^{\mathcal{K}_{i}}(A)\right\rangle_{M_{L}}=\left\langle\prod_{i=1}^{M} W_{n_{i}}^{\mathcal{K}_{i}}(A) \prod_{j=1}^{N} \omega_{K_{j}}(A)\right\rangle .
$$

It appears that a very convenient way of organization of irrep's of SU(2) group is provided by the polynomials $S_{n}(x)$, closely related to the Chebyshev polynomials. $S_{n}(x)$ are defined recursively by the formula

$$
S_{n+2}(x)=x S_{n+1}-S_{n}(x), \quad n=0,1, \ldots,
$$

together with the initial conditions

$$
S_{0}(x)=1, \quad S_{1}(x)=x .
$$


By virtue of (6), $S_{n}(x)$ expresses $n$-th irrep of $\mathrm{SU}(2)$ in terms of powers of the fundamental representation $x$, denoted as $\mathbf{1}$ henceforth. The explicit solution of (6) is

$$
S_{n}(2 \cos \alpha)=\frac{\sin ((n+1) \alpha)}{\sin \alpha}
$$

The skein relations for the fundamental representation $(n=1)$ of $\mathrm{SU}(2)$ are given by the expression

$$
\begin{gathered}
\left.q^{\frac{1}{4}}\langle\ldots\rangle \ldots\right\rangle-q^{-\frac{1}{4}}\langle\ldots\rangle\langle\cdots\rangle=\left(q^{\frac{1}{2}}-q^{-\frac{1}{2}}\right)\langle\ldots|| \cdots\rangle \\
\langle\cdots \mid \pm 1 \cdots\rangle=-q^{ \pm \frac{3}{4}}\langle\cdots \mid 0 \cdots\rangle
\end{gathered}
$$

where the integers in (7b) mean framings, and $q=\exp \frac{2 \pi i}{k}$. Closing the left legs of all the (three) diagrams in (7a) with arcs, as well as the right ones, next applying (7b), and using the property of locality, we obtain

$$
-\left(q-q^{-1}\right)\left\langle W_{\mathbf{1}}^{\bigcirc}(A)\right\rangle=\left(q^{\frac{1}{2}}-q^{-\frac{1}{2}}\right)\left\langle W_{\mathbf{1}}^{\bigcirc}(A)\right\rangle=\left(q^{\frac{1}{2}}-q^{-\frac{1}{2}}\right)\left\langle W_{\mathbf{1}}^{\bigcirc}(A)\right\rangle^{2} .
$$

Hence

$$
\left\langle W_{\mathbf{1}}^{\bigcirc}(A)\right\rangle=-\left(q^{\frac{1}{2}}+q^{-\frac{1}{2}}\right)=-2 \cos \frac{\pi}{k},
$$

and by virtue of the so-called satellite formula

$$
\left\langle W_{n}^{\bigcirc}(A)\right\rangle=S_{n}\left(-2 \cos \frac{\pi}{k}\right)=(-)^{n} \frac{\sin \frac{(n+1) \pi}{k}}{\sin \frac{\pi}{k}}=(-)^{n} \frac{q^{\frac{n+1}{2}}-q^{-\frac{n+1}{2}}}{q^{\frac{1}{2}}-q^{-\frac{1}{2}}} .
$$

We can observe a remarkable property of (8) for $n=k-1$, namely

$$
\left\langle W_{k-1}^{\bigcirc}(A)\right\rangle=0 .
$$

It appears that for any $\mathcal{K}$

$$
\left\langle\cdots W_{k-1}^{\mathcal{K}}(A) \cdots\right\rangle=0 .
$$

In particular, Eq. (10) immediately follows from (9) for any $\mathcal{K}$ that can be unknotted with corresponding skein relations. Thus we can truncate representations of SU(2) above the value $k-2$, and assume

$$
0 \leq n \leq k-2, \quad k=2,3, \ldots
$$

The final explicit form of $\omega_{K}$ for the group $\mathrm{SU}(2)$ is then

$$
\omega_{K}(A)=\sum_{n=0}^{k-2}(-)^{n} \frac{q^{\frac{n+1}{2}}-q^{-\frac{n+1}{2}}}{q^{\frac{1}{2}}-q^{-\frac{1}{2}}} S_{n}\left(W_{1}^{K}(A)\right) .
$$

Strictly speaking, $Z\left(M_{L}\right)$ is invariant with respect to the second Kirby move $\mathrm{K}_{2}$. It means that it is insensitive to the operation of sliding one of its handles over another one. But up to now we have not considered the issue of the determination of normalization. It appears that proper normalization of the partition function $Z\left(M_{L}\right)$ universally follows from the requirement of its invariance with respect to the first Kirby move $K_{1}$. 
The approach proposed above differs from Witten's one [Wit1] in that we explicitly construct the invariant via the cutting and pasting procedure using standard fieldtheoretic tools.

3. Generalization to 4 dimensions. An arbitrary closed connected orientable 4manifold $\mathcal{M}$ can be obtained via surgery in $\mathcal{S}^{3}$ on a special framed link $(L, f)[\mathrm{CdS}]$.

Definition. The special framed link $L$ is a sum of two sorts of knots

$$
L=\bigcup_{i=1}^{N} K_{i} \cup \bigsqcup_{i=1}^{\dot{N}} \dot{K}_{i}
$$

where $\left\{K_{i}\right\}_{i=1}^{N}$ are ordinary knots (corresponding to 2-handles), and $\left\{\dot{K}_{i}\right\}_{i=1}^{\dot{N}}$ are special knots (corresponding to 1-handles). The special knots, denoted with dots, are trivial (with zero framing), and mutually unlinked unknots, and the whole link, when regarded as a description of a 3 -manifold, represents a connected sum of copies of $\mathcal{S}^{1} \times \mathcal{S}^{2}$. The symbol "ப" means the "distant sum" - the components are mutually unlinked.

Now, we introduce the following decomposition (gradation) of $\omega$, defined in (11), into an even $(+)$ and odd $(-)$ parts (integer and half-integer 'spins', respectively)

$$
\omega=\omega^{+}+\omega^{-} \text {. }
$$

Let us denote as $\tilde{K}$ the result of pushing a knot $K$ off itself (missing the rest of the link $L$ ) using the framing $f$ of $K$, whereas as $K_{1} \#_{b} K_{2}$ a (band) connected sum of the two knots $K_{1}, K_{2}$, where $b$ is any band missing the rest of $L$.

Proposition. For arbitrary complex numbers, $a^{+}, a^{-}$, we have the following 'Kirby calculus'

$$
\begin{aligned}
& \left\langle\cdots \alpha_{K_{1}} \cdots \omega_{K_{2}} \cdots\right\rangle=\left\langle\cdots \alpha_{K_{1} \#_{b} \tilde{K}_{2}} \cdots \omega_{K_{2}} \cdots\right\rangle, \\
& \left\langle\cdots \alpha_{K_{1}}^{2} \cdots\left(a^{+} \omega^{+}+a^{-} \omega^{-}\right)_{K_{2}} \cdots\right\rangle=\left\langle\cdots \alpha_{K_{1} \#_{b} \tilde{K}_{2}}^{2} \cdots\left(a^{+} \omega^{+}+a^{-} \omega^{-}\right)_{K_{2}} \cdots\right\rangle,
\end{aligned}
$$

where $\alpha$ is a linear combination of $S_{n}$ with arbitrary coefficients (compare with the definition of $\omega(11))$.

Re mark. The first equality expresses a standard property of $\omega$, whereas the second one follows from the observation that the even element $\alpha^{2}$ respects the gradation in all cablings.

COROLlary. From the Proposition we can derive the following 'Kirby equalities'

$$
\begin{aligned}
\left\langle\cdots \omega_{K_{1}} \cdots \omega_{K_{2}} \cdots\right\rangle & =\left\langle\cdots \omega_{K_{1} \#_{b} \tilde{K}_{2}} \cdots \omega_{K_{2}} \cdots\right\rangle, \\
\left\langle\cdots \omega_{K_{1}}^{+} \cdots \omega_{K_{2}}^{+} \cdots\right\rangle & =\left\langle\cdots \omega_{K_{1} \#_{b} \tilde{K}_{2}}^{+} \cdots \omega_{K_{2}}^{+} \cdots\right\rangle, \\
\left\langle\cdots \omega_{K_{1}}^{+} \cdots \omega_{K_{2}} \cdots\right\rangle & =\left\langle\cdots \omega_{K_{1} \#_{b} \tilde{K}_{2}}^{+} \cdots \omega_{K_{2}} \cdots\right\rangle .
\end{aligned}
$$

The fourth equality,

$$
\left\langle\cdots \omega_{K_{1}} \cdots \omega_{K_{2}}^{+} \cdots\right\rangle=\left\langle\cdots \omega_{K_{1} \#_{b} \tilde{K}_{2}} \cdots \omega_{K_{2}}^{+} \cdots\right\rangle,
$$


is, in general, not true.

Henceforth, $H$ and $\dot{H}$ are two components of the special Hopf link $\mathcal{H}$, ordinary and special one respectively, $\mathcal{H}=H \cup \dot{H}$.

TheOrEm. Let $\nu$ be the nullity of the (extended) linking matrix $\ell k$. Then

$$
I_{k}(\mathcal{M})=\frac{\left\langle\prod_{i=1}^{N} \omega_{K_{i}}^{+} \prod_{i=1}^{\dot{N}} \omega_{\dot{K}_{i}}\right\rangle}{\left\langle\omega_{\bigcirc}^{+}\right\rangle^{\nu}\left\langle\omega_{H}^{+} \omega_{\dot{H}}\right\rangle^{(N+\dot{N}-\nu) / 2}}
$$

is an invariant of the closed, connected, orientable 4-manifold $\mathcal{M}=\mathcal{M}_{L}$, a complex number parametrized by the integer $k$, independent of the choice of the representative $(L, f)$.

Below, we give a list of all the allowable '4-dimensional Kirby moves', so-called $\Gamma$ moves $[\mathrm{CdS}]$ :

(a) sliding one of the special knots over another special one;

(b) sliding one of the ordinary knots over one of the special ones;

(c) sliding one of the ordinary knots over another ordinary one;

(d) introducing or deleting a special Hopf link;

(e) introducing or deleting a trivial unknot;

(f) isotoping the link picture in $\mathcal{S}^{3}$.

Proof. We should show that $I_{k}(\mathcal{M})$ is invariant with respect to all the $\Gamma$-moves. $a_{-}^{-}$, $b$ - and $c$-invariance of $I_{k}(\mathcal{M})$ immediately follows from the Corollary as well as from the invariance of $N, \dot{N}$ and $\nu$. $d$-invariance is a consequence of the following transformation rule of the linking matrix $\ell k$, accompanying the introduction of a special Hopf link $\mathcal{H}$,

$$
\ell k \longrightarrow\left(\begin{array}{ccc}
\ell k & 0 & 0 \\
0 & 0 & 1 \\
0 & 1 & 0
\end{array}\right)
$$

Hence the corresponding shift of the dimension and nullity of $\ell k$

$$
\begin{gathered}
N \longrightarrow N+1 \\
\dot{N} \longrightarrow \dot{N}+1 \\
\nu \longrightarrow \nu,
\end{gathered}
$$

compensates the (factorized out) Kauffman bracket in the numerator. Similarly, e-invariance corresponds to the transformation rule

$$
\ell k \longrightarrow\left(\begin{array}{cc}
\ell k & 0 \\
0 & 0
\end{array}\right)
$$

and consequently the shift

$$
\begin{gathered}
N \longrightarrow N+1 \\
\dot{N} \longrightarrow \dot{N} \\
\nu \longrightarrow \nu+1,
\end{gathered}
$$

also compensates the numerator. $f$-invariance directly follows from fundamental properties of the Kauffman bracket and the linking matrix $\ell k$. 
Rem a r k 1 . The invariant $I_{k}(\mathcal{M})$ possesses the following obvious properties:

1) multiplicativity,

$$
I_{k}(\mathcal{M} \# \mathcal{N})=I_{k}(\mathcal{M}) \cdot I_{k}(\mathcal{N})
$$

2) orientation sensitivity,

$$
I_{k}(\overline{\mathcal{M}})=\overline{I_{k}(\mathcal{M})}
$$

3) normalization,

$$
I_{k}\left(\mathcal{S}^{4}\right)=1
$$

where $\mathcal{M} \# \mathcal{N}$ denotes a connected sum of $\mathcal{M}$ and $\mathcal{N}$.

R e mark 2. Crane and Yetter [CY] have found a 4-dimensional topological invariant, defined via triangulation, which is basically equivalent to ours.

Remark 3. It has been proved that $I_{k}(\mathcal{M})$ is expressible by classical invariants (signature and Euler character) [CKY2], but there is a gap in the proof announced in [CKY1].

4. Final remarks. In the 3 -dimensional case, besides the original RTW invariant, we have some other invariants of the same CS origin, and therefore more or less mutually related. The so-called Turaev-Viro invariant [TV] (the Crane-Yetter invariant, mentioned in Remark 2, is its 4-dimensional counterpart) can be calculated from triangulation of the manifold, and corresponds to the square of the modulus of the RTW invariant. Whereas the Kohno invariant can be calculated from the Heegaard decomposition of the manifold, and is also basically equivalent to the RTW invariant [Bro2]. Perturbative expansion provides us with some further family of invariants, defined for homology spheres, socalled Ohtsuki-Garoufalidis invariants [Oht]. The first perturbative term of this family is the famous Casson(-Walker) invariant, which was originally defined non-perturbatively, and has a surgical description.

In the 4-dimensional situation, we have also a distinct world of 'differentiable invariants', the famous Donaldson invariants and their building block, the Seiberg-Witten invariant [Wit2], mathematically described in this volume. But up to now, it is no clear whether the 'differentiable invariants' are related to the combinatorial ideas presented here.

\section{References}

[Bro1] B. Broda, A surgical invariant of 4-manifolds, Proc. Conf. on Quantum Topology, Kansas 1993, D. N. Yetter (ed.), World Scientific, Singapore, 1994, 45-50.

[Bro2] B. Broda, TQFT versus RCFT: 3-D topological invariants, Modern Phys. Lett. A 10 (1995), 331-336.

[Bro3] B. Broda, Chern-Simons approach to three-manifold invariants, Modern Phys. Lett. A 10 (1995), 487-493.

[CdS] E. César de Sá, A link calculus for 4-manifolds, Topology of low-dimensional manifolds, Proc. Sec. Conf. Sussex, Lecture Notes in Math. 722, Springer, Berlin, 1979, 16-30. 
[CKY1] L. Crane, L. H. Kauffman and D. Yetter, $U_{q}\left(s l_{2}\right)$ Invariants at Principal and Non-principal Roots of Unity, Adv. Appl. Clifford Algebras 3 (1993), 223.

[CKY2] L. Crane, L. H. Kauffman and D. Yetter, On the Classicality of Broda's SU(2) Invariants of 4-Manifolds, Adv. Appl. Clifford Algebras 3 (1993), 223.

[CY] L. Crane and D. Yetter, A categorical construction of $4 d$ topological quantum field theories, Quantum Topology, L. Kauffman and R. Baadhio (eds.), World Scientific, Singapore, 1993.

[Oht] T. Ohtsuki, A polynomial invariant of integral homology 3-spheres, Math. Proc. Cambridge Philos. Soc. 117 (1995), 83-112.

[RT] N. Reshetikhin and V. G. Turaev, Invariants of 3-manifolds via link polynomials and quantum groups, Invent. Math. 103 (1991), 547-597.

[Rol] D. Rolfsen, Knots and Links, Publish or Perish, Wilmington, 1976, Chapt. 9.

[TV] V. G. Turaev and O. Y. Viro, State sum invariants of 3-manifolds and quantum 6j-symbols, Topology 31 (1992), 865-902.

[Wit1] E. Witten, Quantum Field Theory and the Jones polynomial, Comm. Math. Phys. 121 (1989), 351-399.

[Wit2] E. Witten, Monopoles and four-manifolds, Math. Res. Lett. 1 (1994), 769-796. 